\title{
FOURIER ANALYSIS OF SUB-STATIONARY PROCESSES WITH A FINITE MOMENT
}

\author{
BY \\ R. M. DUDLEY
}

Introduction. The purpose of this paper is to study the Fourier transforms of the sample functions $x(t)$ of random processes for which a moment $E|x(t)|^{p}$ is finite and bounded in $t$ for some $p>0$; for example, stationary processes with $E|x(0)|^{p}$ finite. $x(t)$ will not in general approach 0 at $\infty$ and its Fourier transform will not usually be a function, but can be defined as a tempered distribution in Schwartz's theory [8].

The main result is that if $p>1$ the Fourier transform of $x(t)$ is a "Stieltjes integral," i.e. it is obtained from a locally integrable function by differentiating once in the sense of distributions.

Since local properties of the Fourier transform have little to do with local properties of $x(t)$, it is not surprising that the same thing is true if $x(t)$ is replaced by a "random distribution," i.e. a process possibly so irregular that values $x\left(t_{0}\right)$ at points $t_{0}$ are not defined, but with averages " $\int x(t) f(t) d t$ " for smooth functions $f$ defined; we require $p$ th moments of such averages bounded under translation of $f$. It is also possible to replace the real line as domain of values for $t$ by a Euclidean space of arbitrary dimension $k$; here a Stieltjes integral is of the form $\partial^{k} F / \partial t_{1} \cdots \partial t_{k}$ with $F$ locally integrable, and this is the form of the Fourier transforms, again for $p>1$.

The results are stated in detail in $\S 2$. The positive assertions for $p>1$ are proved in $\S \S 4-6$, and the counter-examples for $p \leqq 1$ are given in $\S 7 . \S \S 1$ and 3 deal with random distributions in general. $\$ 8$ shows that the results are easily adapted to the case of processes with nonnegative or discrete parameter or stationary increments.

For stationary Gaussian processes, the results appeared in my Princeton thesis, written with the helpful advice of G. A. Hunt and Edward Nelson. There the methods were based on Ito's "spectral measure," requiring both second moments and strict stationarity of the process or its increments ([4], [5]); here using other methods, essentially the Hausdorff-Young inequality, almost as much is proved from much more general hypotheses.

1. Random distributions. A random distribution, as defined by Gel'fand [1], is a mapping $f \rightarrow L(f)$ of a space $\mathscr{D}=\mathscr{D}\left(R^{k}\right)$, i.e. the space of $C^{\infty}$ functions with

Received by the editors November 6, 1962 and, in revised form, June 22, 1964. 
compact support on a Euclidean space $R^{k}$, into complex-valued random variables (measurable functions) $\omega \rightarrow L(f)(\omega)$ on a probability space $(\Omega, P)$, which is linear and continuous in the following sense: $L(a f+b g)=a L(f)+b L(g)$ with probability one for each $f, g \in \mathscr{D}$ and complex numbers $a, b$, and if $f_{n} \rightarrow 0$ in $\mathscr{D}$, in the usual sense $\left(f_{n}\right.$ all vanish outside some compact set, and their partial derivatives of all orders each converge uniformly to 0 ), then $L\left(f_{n}\right) \rightarrow 0$ in probability (measure).

If $\mathscr{D}$ is replaced by the larger space $\mathscr{S}$ of rapidly decreasing $C^{\infty}$ functions with its usual convergence (see [8, Tome II, Chapitre VII, §3, pp. 89-90]), we shall speak of a random tempered distribution, and in general, if $\mathscr{D}$ is replaced by any topological linear space $\mathscr{T}$, we will call $L$ a random linear mapping on $\mathscr{T}$. Thus what has been called a "weak distribution" on a topological linear space is here a random linear mapping on its dual space.

An ordinary stochastic process $t \rightarrow x(t)(\omega)$ on the real line, if its sample functions are almost all locally integrable, defines a random distribution for $k=1$ by

$$
L_{x}(f)(\omega)=\int_{-\infty}^{\infty} f(t) x(t)(\omega) d t .
$$

A random distribution $L$ is said to be stationary if for any $f_{1}, \cdots, f_{n} \in \mathscr{D}\left(R^{k}\right)$ and $v \in R^{k}$, with $g_{j}(x)=f_{j}(x+v), j=1, \cdots, n$, the joint probability law of $L\left(g_{1}\right), \cdots, L\left(g_{n}\right)$ is the same as that of $L\left(f_{1}\right), \cdots, L\left(f_{n}\right)$. Also, $L$ will be said to have finite $p$ th moments, where $p>0$, if

$$
E|L(f)|^{p}=\int_{\Omega}|L(f)(\omega)|^{p} d P(\omega)<\infty
$$

for all $f \in \mathscr{D}$. The case of stationary random distributions with $k=1, p \geqq 2$, was fully treated by Ito ([4], [5]), and his results carry over easily to all $k>1$, $p \geqq 2$. He showed that there is a measure $\mu$ of polynomial growth at $\infty$ such that for all $f$ and $g \in \mathscr{D}$,

$$
E(L(f) \overline{L(g)})=\int \tilde{f}(\tilde{g})^{-} d \mu,
$$

where $\tilde{f}, \tilde{g}$ are the Fourier transforms of $f, g$. Then from the order of growth of $\mu$ at $\infty$ he determined a number $n$ such that the "sample distributions" $f \rightarrow L(f)(\omega)$, $\omega$ fixed, can be taken to be almost all of the form $d^{n} G_{\omega}(t) / d t^{n}$ (or $\partial^{k n} G_{\omega} / \partial t_{1} \cdots \partial t_{k}^{n}$ for $k>1$ ), where $G_{\omega}$ is a locally integrable function.

This paper is devoted to investigating the sample distributions through their Fourier transforms; the restriction $p \geqq 2$ will be removed and the assumption of stationarity weakened to the following:

DefinItion. A random distribution $L$ is $p$-sub-stationary if for any $f \in \mathscr{D}$ there is a $K>0$ such that $E\left|L\left(f_{v}\right)\right|^{p} \leqq K$ for all $v \in R^{k}$, where $f_{v}(x)=f(x-v)$. 
2. Statement of results. Here is a statement of the main results of this paper; $\tilde{L}_{\omega}$ will denote the Fourier transform of $L_{\omega}$, and on $R^{k}$ we let $x=\left(x_{1}, \cdots, x_{k}\right)$, $d x=d x_{1} \cdots d x_{k}, p>0$.

THEOREM. Let $L=(L, \Omega, P)$ be a p-sub-stationary random distribution. Then there is a mapping $\omega \rightarrow L_{\omega}$ of $\Omega$ into the tempered distributions such that for each $f \in \mathscr{D}, L_{\omega}(f)=L(f)(\omega)$ with probability 1 . If $p>1$, then for each $\omega$ there is a function $F_{\omega}$ such that

$$
\tilde{L}_{\omega}=\frac{\partial^{k} F_{\omega}}{\partial x_{1} \cdots \partial x_{k}}
$$

in the sense of distributions, and

$$
\int_{U}\left|F_{\omega}(x)\right|^{r} d x<\infty
$$

if $0 \leqq r<\infty$ and $U$ is a bounded set. If $p=1$, the above need not be true even for $r=1$, and as $p \rightarrow 0$, to give representations

$$
\tilde{L}_{\omega}=\frac{\partial^{|q|} F_{\omega}}{\partial x_{1}^{q_{1}} \cdots \partial x_{k}^{q_{k}}}
$$

requires in some cases that $|q|=q_{1}+\cdots+q_{k}$ become arbitrarily large.

NoTE. It will be shown in $\S 9$ that, even for $p$ arbitrarily large, the functions $F_{\omega}$ need not be essentially bounded on bounded sets $U$.

The Fourier transforms of the sample functions of an ordinary stationary stochastic process $x(t)(\omega)$ or sequence $x(n)(\omega)$ are not usually functions but more general distributions, no matter how regular $x(t)$ may be; on the other hand, given the restrictions on the growth of $x(t)$ at $\infty$ imposed by laws of large numbers, it is not surprising that its Fourier transform is not too irregular locally.

The theorem applies directly, if crudely, to processes $x(t), t \geqq 0$, after setting $x(t)=0, t<0$, and to sequences $x(n)$ through $L(f)=\Sigma_{n} x(n) f(n)$. For processes with stationary increments it turns out that the Fourier transforms are Stieltjes integrals except at 0 , where they may be one step worse (see $\S 8$ ).

3. Existence theorems for general random distributions. In the general case given an arbitrary random distribution $L$, it is desirable to show first of all that, after a possible alteration of each $L(f)(\omega), f$ fixed, on a set of $P$-measure zero, the functional $f \rightarrow L(f)(\omega)$ for fixed $\omega$ is linear and continuous on $\mathscr{D}$ (is a distribution). This is true (Theorem $\mathrm{C}$ below), but depends partly on the fact that $\mathscr{D}$ is a "nuclear" space. It would not be true, for example, for a Gaussian random linear mapping $L$ on a Hilbert space $(\mathscr{H},\|\|)$ with $E|L(f)|^{2}=\|f\|^{2}$ and $E(L(f))=0$ for all $f \in \mathscr{H}$. 
A random linear mapping which can be altered as described above so as to become linear and continuous for each $\omega$ will be called canonical.

R. A. Minlos [7] has proved that every random linear mapping on a countablynormed nuclear space is canonical. (The terminology will be explained below.) An exposition of Minlos' theorem also appears in Gel'fand and Vilenkin [2]. A very short proof of the theorem has been given by Kolmogorov [11].

A topological linear space $S$ is called nuclear if there is a collection $\mathscr{B}$ of inner products $B($,$) on S$ with the following properties:

(a) $\mathscr{B}$ defines the topology of $S$, i.e. the class of sets

$$
A_{B, \varepsilon}=\{x \in S: B(x, x)<\varepsilon\}, \varepsilon>0, B \in \mathscr{B},
$$

is a base for the neighborhoods of zero in $S$.

(b) For each $B \in \mathscr{B}$ there is a $C \in \mathscr{B}$ such that $B(x, x) \leqq C(x, x)$ for all $x$, and in the Hilbert space which is the completion of $S$ under $C$, on which $B$ is defined by continuity, we have $B(x, x)=C(A x, A x)$ where $A$ is of trace class with respect to $C$. ( $A$ may be assumed self-adjoint (for $C$ ), replacing it by $\sqrt{ }\left(A^{*} A\right)$ if necessary.)

Since on a Hilbert space an operator is nuclear in the sense of [3] if and only if it has finite trace, the above definition is equivalent to Grothendieck's by [3, Chapitre II, Théorème 6, p. 34; Lemme 3, p. 37]. It is known that the spaces arising in theory of distributions such as $\mathscr{E}, \mathscr{E}^{\prime \prime}, \mathscr{D}, \mathscr{D}^{\prime}, \mathscr{S}$, and $\mathscr{S}^{\prime}$ are all nuclear [3, Chapitre II, Théorème 10, p. 55]; those of interest here are $\mathscr{D}$ and $\mathscr{S}$. See also Gel'fand and Vilenkin [2].

A nuclear space is "countably-normed" if the set of inner products in the definition may be chosen countable, and "complete" if it is the intersection of its completions for these inner products. Here is Minlos' theorem:

THEOREM A. Every random linear mapping $L$ on a complete countablynormed nuclear space $S$ is canonical.

The conclusion actually stated by Minlos is that the measure $v$ defined on sets $A$ in $S^{*}$ (the dual of $S$ ) of the form

$$
A=\left\{z \in S^{*}:\left(z\left(f_{1}\right), \cdots, z\left(f_{m}\right)\right) \in B\right\},
$$

where $B$ is a Borel set in complex $m$-space and $f_{1}, \cdots, f_{m} \in S$, by

$$
v(A)_{q}=P\left(\left(L\left(f_{1}\right), \cdots, L\left(f_{m}\right)\right) \in B\right),
$$

which as given is only finitely additive, actually extends to a countably additive measure on $S^{*}$. To prove the conclusion stated above, let $M$ be a countable set dense in $S$ (such a set exists since an operator of trace class on a Hilbert space vanishes on the orthogonal complement of a separable subspace, and we can choose a countable dense set in each of countably many such subspaces). Also 
let $N$ be a countable set dense in the complex numbers. We may assume $M$ is closed under addition and multiplication by numbers in $N$.

Also, $S$ has a translation-invariant metric $\sigma(\sigma(f, g)=\sigma(f-g, 0))$ defining its topology.

For $j, r=1,2, \cdots$, let $A_{j, r}$ be the set of all $\omega \in \Omega$ such that for some $f, g \in M, \sigma(f, g)<1 / j$ and $|L(f)(\omega)-L(g)(\omega)|>1 / r$. Then for each fixed $r$, the intersection of all $A_{j, r}$ has $P$-measure 0 since $v$ has a countably additive extension to $S^{*}$. Thus with probability 1 , the function $f \rightarrow L(f)(\omega)$ on $M$ is uniformly continuous and thus has a continuous extension $L_{\omega}$. Then given $f, g \in S$ and complex numbers $a, b$, let $f_{m} \rightarrow f, g_{m} \rightarrow g, a_{m} \rightarrow a, b_{m} \rightarrow b, f_{m}, g_{m} \in M$, $a_{m}, b_{m} \in N$. With probability $1, L_{\omega}$ is linear on $M$ over $N$. Then since $a_{m} f_{m}+b_{m} g_{m} \in M$ for all $m$,

$$
\begin{aligned}
L_{\omega}(a f+b g) & =\lim L_{\omega}\left(a_{m} f_{m}+b_{m} g_{m}\right) \\
& =\lim \left[a_{m} L_{\omega}\left(f_{m}\right)+b_{m} L_{\omega}\left(g_{m}\right)\right] \\
& =a L_{\omega}(f)+b L_{\omega}(g) .
\end{aligned}
$$

Thus with probability $1, L_{\omega} \in S^{*}$, so of course we may assume $L_{\omega} \in S^{*}$ for all $\omega$. It is also clear that the map $\omega \rightarrow L_{\omega}$ is unique up to alteration on sets of measure 0 .

Theorem A implies directly, since $\mathscr{S}$ is countably-normed, nuclear, and complete:

THEOREM B. Any random tempered distribution is canonical.

This is all we shall use later. However, more general cases seem to be worth mentioning. Let us give the space $\mathscr{M}(\Omega, P)$ of equivalence-classes of measurable functions on a probability space $(\Omega, P)$ the topology of the metric $\rho$ defined by

$$
\rho(f, g)=\inf (\{\varepsilon: P(|(f-g)(\omega)|>\varepsilon)<\varepsilon\}) .
$$

Convergence for this metric is equivalent to convergence in probability. (Note, however, that its topology is not locally convex.) Now, if we eliminate the assumptions that $S$ is countably-normed and complete, but assume that $L$ is not only sequentially continuous but topologically continuous, then $L$ is canonical since it is continuous with respect to some countable set of norms, which may be assumed to define a nuclear space, and $L$ can be extended to the completion of $S$ for these norms by uniform continuity $(\mathscr{M}(\Omega, P)$ is complete for $\rho)$.

On $\mathscr{D}$, sequential continuity suffices:

THEOREM C. Any random distribution is canonical.

This is proved in Gel'fand and Vilenkin [2,p.413], using the fact that the space $\mathscr{D}\left(B_{n}\right)$ of $C^{\infty}$ functions vanishing for $|x| \geqq n$ is countably-normed and nuclear and that $\mathscr{D}$ is the inductive limit of these spaces. 
Of course Theorem B is not a corollary of Theorem C since Theorem B yields $L_{\omega}$ in $\mathscr{S}^{\prime}$.

4. Use of moments. If $(\Omega, P)$ is a probability space and $p>0$, then $\mathscr{L}^{p}(\Omega, P)$ is the linear space of equivalence-classes of measurable functions $f$ on $\Omega$ for which

$$
\left[\int_{\Omega}|f(\omega)|^{p} d P(\omega)\right]^{1 / p}
$$

is finite, with the topology of that norm if $p \geqq 1$, or of the invariant metric given by its $p$ th power if $p<1$.

Now, if $L$ is a random linear mapping from a topological linear space $S$ to $\mathscr{M}(\Omega, P)$ such that for all $f \in S, E|L(f)|^{p}<\infty$, then $L$ defines a linear map of $S$ into $\mathscr{L}^{p}(\Omega, P)$. If $f_{n} \rightarrow f$ in $S$, then $L\left(f_{n}\right) \rightarrow L(f)$ in probability, so by Fatou's lemma

$$
E|L(f)|^{p} \leqq \liminf _{n \rightarrow \infty} E\left|L\left(f_{n}\right)\right|^{p} .
$$

Thus the set $S_{m}$ of all $f \in S$ such that $E|L(f)|^{p} \leqq m$ is sequentially closed in $S$. Thus by a category argument we have:

LEMMA 2. If $S$ is a complete metric linear space and $L$ is a linear mapping of $S$ into $\mathscr{M}(\Omega, P)$ continuous in probability, whose range is contained in $\mathscr{L}^{p}(\Omega, P)$, then $L$ is continuous from $S$ to $\mathscr{L}^{p}(\Omega, P)$.

Now let $L$ be a $p$-sub-stationary random distribution and for $h \in \mathscr{D}$ let $B_{p}(h)=\left(E|L(h)|^{p}\right)^{1 / p}, \quad h_{v}(x)=h(x-v), \quad$ and $\quad A_{p}(h)=\sup _{v} B_{p}\left(h_{v}\right)<\infty$. For $g \in \mathscr{D}$ let

$$
\|g\|_{n}^{2}=\int_{R^{k}} \sum_{|q| \leqq n}\left|D^{q} g\right|^{2} d x,
$$

where $q=\left\langle q_{1}, \cdots, q_{k}\right\rangle,|q|=q_{1}+\cdots+q_{k}, D^{q}=\partial^{|q|} / \partial x_{1}^{q_{1}} \cdots \partial x_{k}^{q_{k}}$. Then $\mathscr{D}\left(B_{2 k}\right)$ has the complete metric

We then have:

$$
\rho(f, g)=\sum_{n=1}^{\infty}\|f-g\|_{n} / 2^{n}\left(1+\|f-g\|_{n}\right) .
$$

Lemma 3. $A_{p}$ is continuous on $\mathscr{D}\left(B_{2 k}\right)$.

Proof. As in Lemma 2 we need only show that the set of $f \in \mathscr{D}\left(B_{2 k}\right)$ such that $A_{p}(f) \leqq 1$ is closed. Suppose $f^{(n)} \rightarrow f$ with $A_{p}(f)>1$ and all $A_{p}\left(f^{(n)}\right) \leqq 1$. Then for some $v, B_{p}\left(f_{v}\right)>1$, but $f_{v}^{(n)} \rightarrow f_{v}$ so $B_{p}\left(f_{v}^{(n)}\right)>1$ for some $n$ by Lemma 2 , a contradiction, q.e.d.

Lemma 4. There exist $K$ and $n$ such that for $f \in \mathscr{D}\left(B_{2 k}\right), A_{p}(f) \leqq K\|f\|_{n}$. 
Proof. By Lemma $3, A_{p}(f)<1$ if $\rho(0, f) \leqq 1 / 2^{n-1}$ for some $n$, and $\rho(0, f) \leqq 1 / 2^{n}+n\|f\|_{n} / 2$ for all $f$. Thus if $\|f\|_{n} \leqq 1 / 2^{n} n, A_{p}(f) \leqq 1$. But $\|f\|_{n}$ and $A_{p}(f)$ are both positively homogeneous functions of $f$. Thus $A_{p}(f) \leqq 2^{n} n\|f\|_{n}$ for all $f \in \mathscr{D}\left(B_{2 k}\right)$, q.e.d.

Now to prove the first assertion of our main theorem the following suffices:

Lemma 5. Any p-sub-stationary random distribution can be extended to a (canonical) tempered random distribution which is also p-sub-stationary.

Proof. Let $g \in \mathscr{D}(R)$ satisfy $g(x)=0,|x| \geqq 1$, and $g(x) \equiv 1-g(x+1)$, $-1 \leqq x \leqq 0$. Let $g_{m}(x)=g(x-m), m=0, \pm 1, \pm 2, \cdots$. Then $\sum_{m=-\infty}^{\infty} g_{m}(x) \equiv 1$, and for each $x$ there are at most two integers $m$ with $g_{m}(x) \neq 0$. We also have on $R^{k}$

$$
\sum_{m_{1}, \cdots, m_{k}=-\infty}^{\infty} g_{m_{1}}\left(x_{1}\right) \cdots g_{m_{k}}\left(x_{k}\right) \equiv 1,
$$

with at most $2^{k}$ individual products nonzero at a given point $\left(x_{1}, \cdots, x_{k}\right)$.

Now let $h_{m}\left(x_{1}, \cdots, x_{k}\right), m=1,2, \cdots$, be an enumeration without repetitions of all functions $g_{m_{1}}\left(x_{1}\right) \cdots \cdot g_{m_{k}}\left(x_{k}\right)$, with functions whose support is nearer the origin being enumerated first. By Lemma 4, there are $n$ and $K>0$ such if $g \in \mathscr{D}$ and the diameter of the support of $g$ is at most $2 k$,

$$
\left(E|L(g)|^{p}\right)^{1 / p} \leqq K\|g\|_{n} .
$$

Let $r=\max (2,2 / p)$ and note that if $f \in \mathscr{S}$ there is a $Q>0$ such that $\left\|f_{v} h_{m}\right\|_{n} \leqq Q / m^{r}, m=1,2, \cdots$, whenever $|v|=\left(v^{2}+\cdots+v_{k}^{2}\right)^{1 / 2} \leqq k$. Let $M \leqq N$ be two positive integers. Then if $p<1$,

$$
\begin{aligned}
E\left|L \sum_{m=M}^{N} f h_{m}\right|^{p} & \leqq E \sum_{m=M}^{N}\left|L\left(f h_{m}\right)\right|^{p} \\
& \leqq \sum_{m=N}^{N}\left(K\left\|f h_{m}\right\|_{n}\right)^{p} \leqq(K Q)^{p} \sum_{m=M}^{N} m^{-2},
\end{aligned}
$$

while if $p \geqq 1$

$$
\begin{aligned}
\left(E\left|L \sum_{m=M}^{N} f h_{m}\right|^{p}\right)^{1 / p} & \leqq \sum_{m=M}^{N}\left(E\left|L\left(f h_{m}\right)^{p}\right|\right)^{1 / p} \\
& \leqq \sum_{m=M}^{N} K\left\|f h_{m}\right\|_{n} \leqq K Q \sum_{m=M}^{N} m^{-2}
\end{aligned}
$$

Thus in either case,

$$
\lim _{M \rightarrow \infty} \sup _{N \geqq M} E\left|L\left(\sum_{m=M}^{N} f h_{m}\right)\right|^{p}=0,
$$

and the series 


$$
\sum_{m=1}^{\infty} L\left(f h_{m}\right)
$$

converges in $p$-norm to a measurable function $L_{1}(f)(\omega)$ on $\Omega$. For any $w \in R^{k}$ we have

$$
E\left|L_{1}\left(f_{w}\right)\right|^{p} \leqq(2 K Q)^{p}
$$

since in the series $\sum_{m=1}^{\infty} L\left(f_{w} h_{m}\right), w$ may be replaced by a vector $v$ from the nearest integral lattice point to $w$, with $|v| \leqq k$. Thus $L_{1}$ is $p$-sub-stationary.

It is clear that $L_{1}(f)=L(f)$ with probability 1 for $f \in \mathscr{D}$, that $L_{1}(a f+b g)=a L_{1}(f)+b L_{1}(g)$ almost surely for any complex $a, b$ and $f, g \in \mathscr{S}$, and that if $f_{j} \rightarrow f$ in $\mathscr{S}, L_{1}\left(f_{j}\right) \rightarrow L_{1}(f)$ in $p$-norm.

Thus $L_{1}$ is a random tempered distribution, canonical by Theorem B; there is a mapping $\omega \rightarrow L_{\omega}$ of $\Omega$ into $\mathscr{S}^{\prime}$ such that for each $f \in \mathscr{S}$,

$$
L_{\omega}(f)=L_{1}(f)(\omega)
$$

for almost all $\omega$. This completes the proof of Lemma 5, and hence of the second sentence of the main theorem.

5. Local properties of Fourier transforms, $p>1$. Since the "sample distributions" of a $p$-sub-stationary random distribution can be taken to be tempered distributions $L_{\omega}$, they have Fourier transforms $\tilde{L}_{\omega}$ also defined as tempered distributions. We now investigate local properties of the $\tilde{L}_{\omega}$.

We shall use Cartesian coordinates $x=\left(x_{1}, \cdots, x_{k}\right)$ on the Euclidean space in which $L$ and $L_{\omega}$ operate, and $t=\left(t_{1}, \cdots, t_{k}\right)$ on the space of the $\tilde{L}_{\omega}$ in duality with $x \cdot t=x_{1} t_{1}+\cdots+x_{k} t_{k}$. Then the Fourier transform $f^{f}$ of a function $f$ in $\mathscr{S}\left(R^{k}\right)$ is defined by

$$
f^{f}(t)=\int_{R^{k}} e^{-2 \pi i(x \cdot t)} f(x) d x,
$$

and the Fourier transform $\tilde{T}$ of a tempered distribution $T$ by $\tilde{T}(f)=T(\tilde{f})$ for all $f \in \mathscr{S}$.

Let $U=U_{Y}$ be a cube $-Y<t_{j}<Y, j=1, \cdots, k$, where $Y>1$. Let $g \in \mathscr{D}\left(R^{k}\right)$ satisfy $g(t)=1$ for $t \in U$. Let $A$ be the largest positive number such that $g$ vanishes outside the cube $C=C(g)=U_{\pi / A}$. For each $\omega, g \tilde{L}_{\omega}$ is the distribution defined by

$$
\left(g \tilde{L}_{\omega}\right)(f)=\tilde{L}_{\omega}(g f) .
$$

Now by the theorem on Fourier series of distributions [8, Tome II, Chapitre VII, §1, Théorème 1, p. 81] we have

$$
\left(g \tilde{L}_{\omega}\right)(f)=\sum_{n} x_{n}(\omega) \int e^{A i n \cdot t} f(t) d t
$$


whenever $f \in \mathscr{D}$ vanishes outside $C$, where $n=\left(n_{1}, \cdots, n_{k}\right)$ runs over all $k$-tuples of integers and

$$
\begin{aligned}
x_{n} & =(A / 2 \pi)^{k} \tilde{L}_{\omega}\left(g e^{-A i n \cdot t}\right) \\
& =(A / 2 \pi)^{k} L_{\omega}\left((\tilde{g})_{n}\right),
\end{aligned}
$$

where $(\tilde{g})_{n}(x)=\tilde{g}(x+A n / 2 \pi)$.

It follows from $p$-sub-stationarity of $L$ and Lemma 5 that whenever $0<r \leqq p$, there is a constant $K>0$ such that

$$
E\left|x_{n}\right|^{r} \leqq\left(E\left|x_{n}\right|^{p}\right)^{r / p}<K
$$

for all $n$. Now if $1<r \leqq p \leqq 2$ let $S$ be any finite set of $k$-tuples $n=\left(n_{1}, \cdots, n_{k}\right)$ of nonzero integers and for any $q>0$ let

$$
Z_{q, S}(\omega)=\left[(2 \pi / A)^{k} \int_{C}\left|\sum_{n \in S} x_{n}(\omega) e^{A i n \cdot t} / p(n)\right|^{q} d t\right]^{r / q},
$$

where $p(n)=n_{1} n_{2} \cdots n_{k} \neq 0, n \in S$. If $1 / r+1 / q=1$, so that $q \geqq 2$, then by the Hausdorff-Young inequality (see Zygmund [9, p. 190]),

$$
Z_{q, S}(\omega) \leqq \sum_{n \in S}\left|x_{n}(\omega) / p(n)\right|^{r}
$$

Thus

$$
E\left(Z_{q, S}(\omega)\right) \leqq \sum_{n \in S} \frac{K}{|p(n)|^{r}}
$$

Now

$$
\sum_{p(n) \neq 0}|p(n)|^{-r}=\left(\sum_{n_{1} \neq 0}\left|n_{1}\right|^{-r}\right)^{k}
$$

is a convergent series, and since in general $\left[\int|f|^{s} d \mu\right]^{1 / s}$ increases with $s$ if $\mu$ has total mass 1 ,

$$
Z_{r, s}(\omega) \leqq Z_{q, s}(\omega)
$$

for all $\omega$. Thus $E\left|Z_{r, s}(\omega)\right|$ approaches 0 as the smallest value of $p(n)$ for $n \in S$ becomes infinite, so the series

$$
\sum_{p(n) \neq 0} x_{n}(\omega) e^{A i n \cdot t} / p(n)
$$

converges in $\mathscr{L}^{r}(\Omega \times C)$, where $\Omega \times C$ has the product measure of $P$ on $\Omega$ and Lebesgue measure on $C$, to a function $G(\omega, t)$. By Fatou's lemma,

$$
\int_{C}|G(\omega, t)|^{q} d t \leqq \liminf _{m \rightarrow \infty} \int\left|\sum_{n \in S_{m}} x_{n}(\omega) e^{A i n \cdot t} / p(n)\right|^{q} d t,
$$


where $S_{m}$ is an increasing sequence of finite sets whose union is the set $P$ of all $n$ with $p(n) \neq 0$. The liminf is at most equal to

so

$$
\left(\sum_{n \in P}\left|\frac{x_{n}(\omega)}{p(n)}\right|^{r}\right)^{q / r}
$$

Thus

$$
\left(\int_{C}|G(\omega, t)|^{q} d t\right)^{r / q} \leqq \sum_{n \in P}\left|\frac{x_{n}(\omega)}{p(n)}\right|^{r} \text {. }
$$

$$
\int_{\Omega}\left(\int_{C}|G(\omega, t)|^{q} d t\right)^{r / q} d P(\omega) \leqq E \sum_{n \in P}\left|\frac{x_{n}(\omega)}{p(n)}\right|^{r}<\infty .
$$

Hence for almost all $\omega$,

$$
\int_{C}|G(\omega, t)|^{q} d t<\infty .
$$

Since $r$ may be arbitrarily near $1, q$ may be arbitrarily large.

Let $G_{\omega}(t)=G(\omega, t)$. Then almost every $G_{\omega}$ has a Fourier series. On the other hand the series

$$
\sum_{n \in P}\left|\frac{x_{n}(\omega)}{p(n)}\right|^{r}
$$

converges for almost all $\omega$, and

$$
\sum_{n \in P} x_{n}(\omega) e^{A i n \cdot t} / p(n)
$$

converges in $\mathscr{L}^{q}(C)$, and hence in $\mathscr{L}^{2}(C)$, for all such $\omega$. Thus for almost all $\omega$, this series is the Fourier series of $G_{\omega}$.

Now let $J$ be any subset of the finite set $(1,2, \cdots, k)$, and let $B_{J}$ be the set of all $n=\left(n_{1}, \cdots, n_{k}\right)$ such that $n_{j} \neq 0$ if and only if $j \in J$. It can be proved just as above that for almost all $\omega$ the series

$$
\sum_{n \in B_{J}} x_{n}(\omega) e^{A i n \cdot t} \mid \prod_{j \in J} n_{j}
$$

is the Fourier series of a function $G_{J, \omega}$ such that $\int_{C}\left|G_{J, \omega}(t)\right|^{q} d t$ is finite for all positive $q$. This is also true of the function

and thus we have

$$
H_{J, \omega}=\left(\prod_{j \xi J} t_{j}\right) G_{J, \omega},
$$

$$
\begin{aligned}
\left(g \tilde{L}_{\omega}\right)(f) & =\left[\sum_{n} x_{n}(\omega) e^{\operatorname{Ain} \cdot t}\right](f) \\
& =\left[\partial^{k} \sum_{J} H_{J, \omega} / \partial t_{1} \cdots \partial t_{k}\right](f),
\end{aligned}
$$


where the sum and partial derivative are taken in the sense of distributions, $J$ runs over all subsets of $(1,2, \cdots, k)$ including the empty set (a product over the empty set, when $J$ is all of $(1,2, \cdots, k)$, is defined to be 1$)$, and $f \in \mathscr{D}$ has support in $C$. If $f$ vanishes outside $U, g \tilde{L}_{\omega}$ may be replaced by $\tilde{L}_{\omega}$ since $g \equiv 1$ on $U$. Let $H_{\omega}$ $=\Sigma_{\mathrm{J}} H_{J, \omega}$, defined on $C$. Of course these functions depend on $g$, and we now make this explicit by letting $H_{\omega}=H_{\omega, g}$.

6. From local to global representation. To finish the proof for $p>1$ we need only remove the dependence of $H_{\omega, g}$ on $g$.

Let $f$ be a $C^{\infty}$ function on the real line with $f(s)=0$ for $|s| \geqq 1$ and

$$
\int_{-1}^{1} f(s) d s=1
$$

For any distribution $T$ on $R^{k}$ and $j=1, \cdots, k$, let $A_{j} T$ be the distribution on $R^{k-1}$ defined by

$$
\left(A_{j} T\right)(g)=T\left(f\left(t_{j}\right) g\left(t_{1}, \cdots, t_{j-1}, t_{j+1}, \cdots, t_{k}\right)\right) .
$$

Let $I_{j} T$ be the distribution on $R^{k}$, independent of $t_{j}$, defined by $A_{j} T$, i.e.

$$
\left(I_{j} T\right)(h)=\left(A_{j} T\right)\left(\int_{-\infty}^{\infty} h\left(t_{1}, \cdots, t_{k}\right) d t_{j}\right) .
$$

If $T$ is a function $F$ with $|F|^{q}$ locally integrable, $q>1$, then

$$
I_{j} F\left(t_{1}, \cdots, t_{k}\right)=\int_{-1}^{1} f\left(t_{j}\right) F\left(t_{1}, \cdots, t_{k}\right) d t_{j},
$$

so that $\left|I_{j} F\right|^{q}$ is also locally integrable.

Now for $t \in C(g)$ let

$$
F_{\omega, g}(t)=\left[\left(\prod_{j=1}^{k}\left(1-I_{j}\right)\right) H_{\omega, g}\right](t) .
$$

Then $D^{(k)}\left(F_{\omega, g}-H_{\omega, g}\right)=0$ as a distribution on $C(g)$, where $D^{(k)}=\partial^{k} / \partial t_{1} \cdots \partial t_{k}$, since $\partial\left(I_{j} H\right) / \partial t_{j}=0$ for any $j$ and $H=H_{\omega, g}$. We want to show that $F_{\omega, g}(t)$ for $t$ in a cube $U_{Y}$ on which $g \equiv 1$ is actually independent of $g$. For this we shall use:

LEMMA 6. If $F$ is an integrable function on a cube $U=U_{Y}, Y>1$, then the following three assertions are equivalent:

(a) $D^{(k)} F=0$ on $U$ as a distribution, i.e. if $f \in \mathscr{D}$ vanishes outside $U$,

$$
(-1)^{k} \int_{U} F D^{(k)} f d t=0
$$

(b) There exist functions $F_{1}, \cdots, F_{k}$ integrable on $U$ with $F_{j}$ independent of $x_{j}, j=1, \cdots, k$, and $F=F_{1}+\cdots+F_{k}$ on $U$. 
(c)

$$
\left[\prod_{j=1}^{k}\left(1-I_{j}\right)\right] F=0 \text { on } U
$$

Proof. It is clear that (b) is equivalent to (c). (b) implies (a) since $\partial F_{j} / \partial t_{j}=0$ for each $j$. Now, it is easy to see that the operations $I_{j}$ and $\partial / \partial t_{i}$ commute for $i \neq j$ and that if $\partial S / \partial t_{j}=0$ on $U$ for any $j, I_{j} S=S$. Thus if $D^{(k)} T=0$ on $U$, $\left(1-I_{1}\right) \partial^{k-1} T / \partial t_{2} \cdots \partial t_{k}=\partial^{k-1}\left(1-I_{1}\right) T / \partial t_{2} \cdots \partial t_{k}=0$ on $U$, and we can replace each $\partial / \partial t_{j}$ by $1-I_{j}$ successively and infer (b), q.e.d.

Now let $g, h \in \mathscr{D}\left(R^{k}\right)$ satisfy $g(t) \equiv h(t) \equiv 1, t \in U_{Y}$. Then on $U_{Y}$

$$
D^{(k)} H_{\omega, g}=\tilde{L}_{\omega}=D^{(k)} H_{\omega, h} .
$$

Thus by Lemma 6

$$
F_{\omega, g}-F_{\omega, h}=\left[\prod_{j=1}^{k}\left(1-I_{j}\right)\right]\left(H_{\omega, g}-H_{\omega, h}\right)=0
$$

on $U_{Y}$. Hence a function $F_{\omega}$ is uniquely defined by $F_{\omega}(t)=F_{\omega, g}(t)$ if $t \in U_{Y}$ and $g \equiv 1$ on $U_{Y}$, and $D^{(k)} F_{\omega}=\tilde{L}_{\omega}$ (everywhere). This completes the proof of the part of the theorem concerning $p>1$, since $\left|F_{\omega}\right|^{q}$ is locally integrable for all positive $q$.

7. Counter-examples for $p \leqq 1$. These will be given only for $k=1$, although it is easy to construct similar examples in higher dimensions.

For $0<\beta<1$ let $\mu_{\beta}$ be the probability measure on the real line with

$$
\mu_{\beta}(x: x \leqq a)=1-a^{-\beta}, \quad a \geqq 1 .
$$

Then let $L(\beta)$ be the random distribution defined by

$$
\sum_{n=-\infty}^{\infty} x_{n} \delta(n+r)
$$

where the $x_{n}$ are independent random variables each with distribution $\mu_{\beta}, r$ is uniformly distributed in the interval $[0,1]$ independently of the $x_{n}$, and $\delta$ is the unit mass at 0 . Thus

$$
L(\beta)(f)(\omega)=\sum_{n=-\infty}^{\infty} x_{n}(\omega) f(n+r(\omega)) .
$$

It is clear that each $L(\beta)$ is stationary and is $p$-sub-stationary if and only if $p<\beta$. Now, almost all the Fourier transforms $L(\beta)_{\omega}^{\sim}$ given by the first part of our theorem are equal to the distributions represented by the series

$$
\sum_{n=-\infty}^{\infty} x_{n}(\omega) \exp (2 \pi i(n+r(\omega)) t)
$$

convergent in the topology of distributions on the line. Thus if $L(B)_{\infty}^{\sim}$ is, with positive probability, locally of the form $d F_{\omega} / d t$, with $F_{\omega}$ locally integrable, then 
so is $\exp (-2 \pi \operatorname{ir}(\omega) t) L(\beta)_{\omega}^{\sim}$. Then $x_{n}(\omega) / n$, is with positive probability, a bounded sequence of real numbers for $n \neq 0$. But for any $K>0$ and $\beta<1$ the series $\sum_{n=-\infty}^{\infty} \mu_{\beta}(x: x \geqq K n)$ is divergent, so that by the zero-one law $x_{n} / n$ is almost never bounded for $n \neq 0$.

Similarly, whenever $L(\beta)_{\omega}^{\sim}$ is locally of the form $d^{m} F_{\omega} / d t^{m}$, then $\sum_{n \neq 0} x_{n} e^{2 \pi i n t} / n^{m}$ is locally integrable (being $f+g F_{\omega}$ with $f$ and $g C^{\infty}$ ), so that $x_{n} / n^{m}, n \neq 0$, is a bounded sequence. Now for $\beta<1$ we have $\mu_{\beta}\left(x: x \geqq K n^{m}\right)=K^{-\beta} n^{-m \beta}$ for $K>0$, so that the sum over $n \neq 0$ of these numbers converges if and only if $\beta>1 / m$. Thus as $\beta \rightarrow 0$ we must let $m \rightarrow \infty$ to obtain a local representation $\tilde{L}_{\omega}=d^{m} F_{\omega} / d t^{m}$. However, such a representation exists for any $p$-sub-stationary random distribution, and an upper bound for $m$ can be determined from the order of growth of the distributions $L_{\omega}$ at $\infty$, which in turn can be bounded in terms of $p$ as in $\$ \S 4-5$.

For $p=1$, we shall use independent random variables with different distributions: let $x_{n}=n \cdot \log (n)$ with probability $1 / n \cdot \log (n), x_{n}=0$ otherwise, $n \geqq 1$. Then $E\left(\left|x_{n}\right|\right)=1$ for all $n$, so $L=\sum_{n=1}^{\infty} x_{n} \delta(x-n)$ is 1 -sub-stationary. Then since the sum of $1 / n \cdot \log (n)$ diverges, $x_{n} / n=\log n$ infinitely often with probability 1 . Since $x_{n} / n$ is almost never bounded, $\widetilde{L}_{\omega}$ is almost never of the form $d F_{\omega} / d t$ with $F_{\omega}$ locally integrable. This completes the proof of the theorem stated in $\S 2$.

Now we show that the $x_{n}$ must have different distributions to yield a counterexample as above for $p=1$, even if they are not independent:

THEOREM. Suppose $x_{n}, n=0, \pm 1, \pm 2, \cdots$, are complex-valued random variables on a probability space $(\Omega, P)$, all having the same probability distribution $\mu$ on the space $C$ of complex numbers with

$$
E(|x|)=\int_{C}|z| d \mu(z)<\infty .
$$

Then for almost all $\omega \in \Omega$,

converges.

$$
\sum_{n \neq 0}\left|\frac{x_{n}(\omega)}{n}\right|^{2}
$$

Proof. Clearly we may assume that the $x_{n}$ are all real-valued. We have

$$
\begin{aligned}
\sum_{n=1}^{\infty} P\left(\left|x_{n}\right| \geqq n\right) & =\sum_{n=1}^{\infty} P\left(\left|x_{1}\right| \geqq n\right)=\sum_{n=1}^{\infty} n P\left(n \leqq\left|x_{1}\right|<n+1\right) \\
& \leqq E\left(\left|x_{1}\right|\right)<\infty .
\end{aligned}
$$

Thus if

$$
\begin{aligned}
& y_{n}=x_{n} \text { for }\left|x_{n}\right|<n \text {, } \\
& =0 \text { otherwise, }
\end{aligned}
$$


then almost surely $y_{n}=x_{n}$ for $n$ large enough. Then for any integer $N \geqq 1$,

$$
\begin{aligned}
E\left(\sum_{n=1}^{N}\left(y_{n} / n\right)^{2}\right) & =\sum_{n=1}^{N} \frac{1}{n^{2}} \int_{-n}^{n} x^{2} d \mu(x) \leqq \int_{1}^{\infty} \frac{4}{y^{2}}\left[\int_{-y}^{y} x^{2} d \mu(x)\right] d y \\
& =\int_{-\infty}^{\infty}\left[\int_{\min (1,|x|)} 4 d y / y^{2}\right] x^{2} d \mu(x) \leqq \int_{-\infty}^{\infty} 4|x| d \mu(x)
\end{aligned}
$$

Thus, using also a similar argument for negative $n$,

$$
E\left(\sum_{n \neq 0}\left(y_{n} / n\right)^{2}\right)<\infty
$$

so $\sum_{n=0}\left(x_{n} / n\right)^{2}$ is almost surely finite, q.e.d.

COROLlaRY. Under the hypotheses of the Theorem,

$$
\sum_{n \neq 0}\left[x_{n}(\omega) / n\right] \exp (2 \pi i n \lambda)
$$

is for almost all fixed $\omega$ the Fourier series of a periodic function $F_{\omega}(\lambda)$ of period 1 with

$$
\int_{0}^{1}\left|F_{\omega}(\lambda)\right|^{2} d \lambda<\infty
$$

We then have

$$
\sum_{n \neq 0} x_{n}(\omega) \exp (2 \pi i n \lambda)=d F_{\omega}(\lambda) / d \lambda
$$

as distributions. A nonzero constant cannot be the derivative of a periodic distribution, but we have

$$
\sum x_{n}(\omega) \exp (2 \pi i n \lambda)=d G_{\omega}(\lambda) / d \lambda
$$

where $G_{\omega}(\lambda)=F_{\omega}(\lambda)+\lambda x_{0}(\omega)$,

In connection with the Corollary, there is a logical point perhaps worth mentioning. Given $(\Omega, P)$ and the $x_{n}(\omega)$ we construct the functions $F_{\omega}()$ on $R$ which may be regarded as making up one function $(\omega, \lambda) \rightarrow F_{\omega}(\lambda)$ on the product space $\Omega \times R$. So, it is assumed that fixing $\omega$ does not restrict $\lambda$. Note, however, that the random variables $x_{n}=\exp (-2 \pi i n \lambda)$ on $0 \leqq \lambda \leqq 1$ with Lebesgue measure are integrable with the same distribution, while $\sum_{n \neq 0} 1 / n$ is not convergent.

A detailed study of random Fourier series with independent coefficients has been made by Kahane [10], and we mention it here for the sake of the interested reader although it has only a small overlap with this paper. 
8. Restricted parameter and stationary increments. For $k=1$, an ordinary one-sided stochastic process $y(t), t \geqq 0$, with $E|y(t)|^{p}$ bounded for some $p>0$, can obviously be extended to a $p$-sub-stationary process by setting $y(t)=0$ or $y(t)=y(-t)$ for $t<0$. Also a sequence $\left\{y_{n}\right\}$ with $E\left|y_{n}\right|^{p}<K<\infty$ for all $n$ defines the $p$-sub-stationary random distribution $\sum_{n} y_{n} \delta(x-n)$. Thus the Theorem can be applied to such processes, quite appropriately to sequences since the method of proof uses them.

A random distribution $L$ on $\mathscr{D}(R)$ is said to have stationary increments if the random distribution $d L / d t$ defined by $(d L / d t)(f)=L(-d f / d t)$ is stationary (see [4]). This coincides with the usual definition for ordinary processes with locally integrable sample functions. If there is $p>0$ such that $E|L(f)|^{p}<\infty$ for all $f \in \mathscr{D}$, then this is also true of $d L / d t$, so $d L / d t$ is $p$-sub-stationary, and the distributions $(d L / d t)_{\omega}=d L_{\omega} / d t$ are tempered. If $p>1$, there are locally integrable functions $F_{\omega}(x)$ such that $(d L / d t)_{\omega}^{\tilde{\omega}}(f)=\tilde{L}_{\omega}(2 \pi i x f(x))=\left(d F_{\omega} / d x\right)(f)$ for all $f \in \mathscr{S}$, so that $\tilde{L}_{\omega}=(1 / 2 \pi i x)\left(d F_{\omega} / a x\right)$ except near $x=0$. Thus $\tilde{L}_{\omega}$ is the first derivative of a locally-summable function except near $x=0$, where a second derivative may be required. Processes with stationary increments on a non negative parameter range can be treated as before; for a discrete process $\left\{y_{n}, n \geqq 0\right\}$ the random distribution $L=S \sum_{n} y_{n} \delta(x-n)$, where for any distribution $T, S T(x)=T(x+1)-T(x)$, is $p$-sub-stationary if $E\left|y_{0}\right|^{p}$ is finite, and so $\tilde{L}_{\omega}=\left(1 /\left(1-e^{2 \pi i t}\right)\right)\left(d F_{\omega} / d t\right)$ except where $t$ is an integer.

9. $F_{\omega}$ need not be locally bounded. Here is an example showing that the functions $F_{\omega}$ given for $p>1$ by our main theorem need not be essentially bounded on bounded sets. Let $k=1$ and let $L^{q}$ be the random distribution defined by a process $x(t)(\omega)$ with

$$
\begin{array}{ll}
x(t)=x(t)(\omega)=1 & \text { for } t \geqq 0, \\
x(t)=x(t)(\omega)=0 & \text { for } t<0 .
\end{array}
$$

Then $d x(t) / d t=\delta(t)$, so for the Fourier transform distribution $\tilde{x}(\lambda)$ we have $(2 \pi i \lambda) \tilde{x}(\lambda)=1$ (see Schwartz [8, Tome II, Chapitre VII, §8, Théorème XV, p. 124]). Thus

$$
\tilde{x}(\lambda)=c \delta(\lambda)+[1 / 2 \pi i \lambda]
$$

for some $c$ (actually $1 / 2$ ), where $[1 / 2 \pi i \lambda]$ is the distribution defined by

$$
[1 / 2 \pi i \lambda](g)=\lim _{\varepsilon \downarrow 0} \int_{|\lambda| \geqq \varepsilon}(g(\lambda) / 2 \pi i \lambda) d \lambda, \quad g \in \mathscr{D}
$$

(see Schwartz [8, Tome I, Chapitre V, §4, Théorème VII, p. 123]). Thus if $\tilde{x}=d F(\lambda) / d \lambda, F(\lambda)=\log (|\lambda|)+h(\lambda)$ where $h$ is a bounded function. Thus $F$ is not locally essentially bounded (near 0 ), although of course it is locally in $L^{r}$ for all finite $r$. 


\section{REFERENCES}

1. I. M. Gel'fand, Generalized random processes, Dokl. Akad. Nauk SSSR 100 (1955), 853-856. (Russian)

2. I. M. Gel'fand and N. Ja. Vilenkin, Generalized functions, No. 4, Some applications of harmonic analysis. Equipped Hilbert spaces, Fizmatgiz, Moscow, 1961. (Russian)

3. A. Grothendieck, Produits tensoriels topologiques et espaces nucléaires, Mem. Amer. Math. Soc. No. 16 (1955).

4. K. Ito, Stationary random distributions, Mem. Coll. Sci. Univ. Kyoto Ser. A Math. 28 (1954), 209-223.

5. - Isotropic random current, Proc. Third Berkeley Symposium on Mathematical Statistics and Probability (1954/55), pp. 125-132, Univ. California Press, Berkeley, Calif., 1956.

6. M. Loève, Probability theory, 2nd ed., Van Nostrand, Princeton, N. J., 1960.

7. R. A. Minlos, Generalized random processes and their extension to measures, Trudy Moskov. Mat. Obšč. 8 (1959), 497-518. (Russian) (1).

8. L. Schwartz, Théorie des distributions, 2nd ed., Tome I, Hermann, Paris, 1957; Tome II, Hermann, Paris, 1959.

9. A. Zygmund, Trigonometrical series, reprint, Chelsea, New York, 1952.

10. J.-P. Kahane, Propriétés locales des fonctions à séries de Fourier aléatoires, Studia Math. 19 (1960), 1-25.

11. A. N. Kolmogorov, Note to the papers of R. A. Minlos and V. Sazonov, Theor. Probability Appl. 4 (1959), 221-223.

\section{UNIVERSITY OF CALIFORNIA, BERKELEY, CALIForNiA}

(1) Translation of the above article, to appear in Selected translations in mathematical statistics and probability, Amer. Math. Soc., Providence, R. I. 